\title{
Kernos
}

Revue internationale et pluridisciplinaire de religion grecque antique

4 | 1991

Varia

\section{Le traité de Porphyre contre les Chrétiens}

L'état de la question

Pier Franco Beatrice

\section{(2) OpenEdition}

9 Journals

Édition électronique

URL : http://journals.openedition.org/kernos/295

DOI : $10.4000 /$ kernos.295

ISSN : 2034-7871

Éditeur

Centre international d'étude de la religion grecque antique

Édition imprimée

Date de publication : 1 janvier 1991

Pagination : 119-138

ISSN : 0776-3824

\section{Référence électronique}

Pier Franco Beatrice, «Le traité de Porphyre contre les Chrétiens », Kernos [En ligne], 4 | 1991, mis en ligne le 11 mars 2011, consulté le 01 mai 2019. URL : http://journals.openedition.org/kernos/295 ; DOI : 10.4000/kernos.295 
Kernos, 4 (1991), p. 119-138.

\section{LE TRAITÉ DE PORPHYRE CONTRE LES CHRÉTIENS. L'ÉTAT DE LA QUESTION}

\section{De l'Antiquité au Moyen Âge}

Le traité que le philosophe grec néoplatonicien Porphyre a écrit contre les Chrétiens est un objet mystérieux.

En effet, de ce traité on ne connaît avec précision ni la date de composition, ni la structure, ni le contenu complet. Nous savons seulement avec certitude qu'il fut écrit en Sicile après l'an 271 après J.C. ${ }^{1}$ et qu'il comptait en tout quinze livres ${ }^{2}$.

Le sort particulier qui revint à cet ouvrage déjà dès la fín de l'Antiquité, explique une situation aussi singulière. Comme cet ouvrage fut considéré pour ce qu'il était et pour ce qu'il voulait être effectivement, c'est-à-dire une violente attaque, une critique impitoyable portées contre le cœur même de la nouvelle religion chrétienne, il fut aussitôt censuré par l'autorité impériale devenue ouvertement philochrétienne avec Constantin.

L'indignation déclenchée par la rude polémique antichrétienne de Porphyre parmi les chrétiens cultivés de son temps, comme Méthode d'Olympe ${ }^{3}$ et Eusèbe de Césarée ${ }^{4}$, amena Constantin à ordonner l'anéantissement du traité porphyrien à cause de son contenu scélérat.

1 EusÈbe de CÉSARÉe, Hist. Eccl., VI, 19, 2 affirme que PORPHyre écrivit cet ouvrage en Sicile. Or nous savons de PORPHYre lui-même qu'il avait quitté Rome pour la Sicile en 268 (Vita Plotini, 6, 1-3). En outre, dans le traité antichrétien, il s'était servi de l'Histoire d'Alexandrie de CALlinicus SuTorius qui, à son tour, n'a pas été composée avant 270. De toutes ces données, Alan

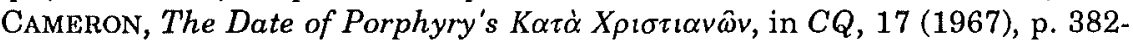
384 (réimprimé dans le volume Literature and Society in the Early Byzantine World, London, 1985, n. X), déduit justement que le terminus post quem se situe vers l'an 271.

2 Cette notice provient de la Souda, s.v. Пopழúpıs, éd. A. Adler, pars IV, p. 178 :

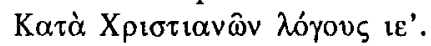

3 Selon JÉrôme, Epist., 70, 3 ad Magnum (CSEL 54, 703), la première réponse chrétienne à PORPHYRe est due à MÉTHOde. Voir aussi Apol. adv. Rufinum, II, 33; De viris inl., 83; Epist., 48, 13; Com. Dan., Prol.

4 D'après JÉRÔME, De viris inl., 81, 3, EusÈBE est l'auteur d'un traité (perdu) Contre Porphyre en 25 livres. 
À ce sujet, Constantin, dans son fameux édit de l'an 333 contre Arius, s'exprime en ces termes:

...Porphyre, l'ennemi de la véritable piété, pour avoir composé plusieurs écrits scélérats contre le culte de Dieu, a trouvé son juste salaire, à savoir un éternel déshonneur, la perte totale de sa réputation et l'anéantissement de ses ouvrages impies.... 5

Cette décision de Constantin marque un changement radical dans le sens qu'il s'agit ici du premier acte de censure des livres opéré par l'État en faveur de l'Église chrétienne ${ }^{6}$. Toutefois, elle n'eut pas l'effet désiré, si l'on pense que des ouvrages massifs furent encore consacrés à la réfutation de Porphyre, comme celui d'Apollinaire de Laodicée, en trente livres, écrit vers l'an $370^{7}$ ou bien la réponse de l'arien Philostorge, qui remonte à l'an $420^{8}$.

Ceci signifie que le traité antichrétien de Porphyre était encore en circulation dans les premières décennies du Ve siècle. Cela n'est pas surprenant. Grégoire de Nazianze, vers 364 , affirme que les païens se complaisent des mensonges de Porphyre ${ }^{9}$, tandis que le rhéteur païen Libanius évoque le vieux de Tyr, c'est-à-dire Porphyre, pour exalter l'ouvrage antichrétien de Julien l'Apostat ${ }^{10}$. Quelques années plus tard, vers 380, Jean Chrysostome atteste explicitement que les écrits des philosophes et des orateurs païens qui avaient été dirigés contre le

5 Le texte grec est reproduit par les historiens ecclésiastiques SocRaTE, I, 9, 30 et GÉLASE, II, 36, 1. Voir l'édition de H.G. OPITZ, Urkunden zur Geschichte des arianischen Streites, Berlin, 1934-1935, Urk. 33.

6 C'est une expression d'Adolf von Harnack, Porphyrius 'Gegen die Christen', 15 Bücher-Zeugnisse, Fragmente und Referate (Abhandlungen d. kgl. preuss. Akad. d. Wiss., Phil.-hist. Kl., Berlin, 1916, 1), Zeugnis, IX, p. 31: «Die Verfügung ist das erste staatliche Bücherverbot im Interesse der Kirche». Sur ce sujet on peut beaucoup apprendre dans l'œuvre savante de W. SPEYER, Büchervernichtung und Zensur des Geistes bei Heiden, Juden und Christen, Stuttgart, 1981 (Bibliothek des Buchwesens, Band 7), en particulier les p. 134 sq.

7 H. Lietzmann, Apollinaris von Laodicea und seine Schule, Tübingen, 1904 (réimpr. Hildesheim, 1970), p. 150 et 265-267.

8 Voir l'édition de J. BiDEz dans GCS, 21, Leipzig, 1913, p. 130.

9 GRÉGOIRE DE NAZIANZE, Orat. c. Jul., V, 41 (SCh, 309, p. 378).

10 LiBAnius, Orat., XVIII, 178-179. Ce texte est cité par SocRATE, Hist. Eccl., III, 23 $(P G, 67,437)$. 
christianisme "s'ils se sont conservés en quelque endroit, c'est chez des Chrétiens qu'on peut les trouver" ${ }^{11}$.

Il serait facile de multiplier les témoignages dispersés dans les écrits chrétiens de l'époque, de Diodore de Tarse ${ }^{12}$ et Némésius $d^{\prime}$ Émèse ${ }^{13}$ jusqu'à l'historien Socrate ${ }^{14}$ et à Théodoret de Cyr $^{15}$. Mais ce qui nous intéresse maintenant c'est que Constantin se soit servi du nom de Porphyre pour stigmatiser et condamner l'hérésie d'Arius à tel point que les disciples de ce dernier furent gratifiés du surnom infamant de «Porphyriens» 16 !

De la même manière, le nom de Porphyre apparaît dans l'édit promulgué par Théodose II et Valentinien III le 17 février 448 pour condamner deux évêques chrétiens, Nestorius et Irénée de Tyr ${ }^{17}$. Dans cet édit, les empereurs chrétiens ordonnent de brûler les livres antichrétiens de Porphyre afin d'empêcher que «les ouvrages susceptibles de mettre Dieu en colère et de nuire aux âmes viennent même jusqu'aux oreilles des hommes» 18 .

On peut dire que, dès ce moment, le sort définitif du traité porphyrien est signé pour toujours. Pour la législation impériale ultérieure, l'édit de Théodose devient normatif : en effet les Préfets y recourent explicitement dans leurs instructions du 18 avril de la même année $448^{19}$ et de

11 De S. Babyla, 11 (SCh, 362, p. 106). Voir, à ce propos, M.A. SchATKIN, Saint John Chrysostom Apologist, Washington, 1985 (The Fathers of the Church, 73), p. 17-23.

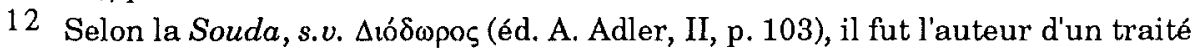
Contre Porphyre sur les animaux et les sacrifices.

13 De natura hominis, 3 (éd. M. Morani, Leipzig, 1987, p. 42).

14 Hist. Eccl., III, 23 (PG, 67, 440 sq.).

15 Thérap., II, 43-44 (SCh, 57, p. 150); VII, 36 (p. 305); XII, 96 (p. 446). L'écrivain syriaque EBED-JESU, dans le Catalogus librorum ecclesiasticorum, composé vers l'an 1200, mentionne un commentaire de THÉODORE DE MOPSUESTE sur l'Ecclésiaste plein de malédictions contre PoRPhYRE (J.S. AssEMANI, Bibliotheca Orientalis Clementino-Vaticana, IIV/1, Roma, 1725, p. 32).

16 Voir encore la Urkunde 33 de H.G. OpITz, citée supra, n. 5. Cette loi de Constantin est mentionnée pour la première fois par ATHANASE D'ALEXANDRIE, Hist. Arianorum ad monachos, $50(P G, 25,753)$ et ensuite par THÉodosE II dans une loi de l'an 435 (Cod. Just., I, 5, 6).

17 Voir le texte grec dans les Acta Conciliorum Oecumenicorum de E. SchwarTZ, I/1, 4, 66 (= Cod. Just. I, 1, 3).

18 Traduction et commentaire chez P. DE LABRIOLLE, La réaction paierine. Etude sur la polémique antichrétienne du Ier au VIe siècle, Paris, 1934, p. 243.

$19 A C O, \mathrm{I} / 1,4,67$. 
même, un siècle plus tard, en 536, l'empereur Justinien dans son édit contre Anthime, Sévère et les autres ${ }^{20}$.

De cette façon, on explique que se soit perdu pour toujours l'ouvrage antichrétien de Porphyre, mais le souvenir historique de son existence ne s'effaça pas du tout.

Encore au Xe siècle, Théophylacte pouvait citer, par tradition indirecte, une interprétation porphyrienne du prologue de l'évangile de $J_{\text {Jean }}{ }^{21}$, pour ne pas parler du lexique byzantin, la Souda, que nous avons déjà mentionné.

Dans l'Occident latin médiéval, le Géographe anonyme de Ravenne, qui écrit au VIIe siècle, définit Porphyre comme miserum et nefandissimum ${ }^{22}$, tandis que, au XIIe siècle, Jean de Salisbury savait, pour l'avoir lu dans le De Civitate Dei d'Augustin, que Porphyre avait été catholicae veritatis pessimus impugnator ${ }^{23}$.

\section{Les premiers recueils des fragments à l'époque moderne}

Après le long silence médiéval, on ne parle de nouveau du traité antichrétien de Porphyre qu'au XVIIe siècle, et naturellement dans un cercle restreint d'érudits.

Cet ouvrage de Porphyre avait certainement éveillé la savante curiosité de l'humaniste Hugo Grotius. Il résulte en effet d'un manuscrit de la bibliothèque de Carpentras qu'il avait commencé à recueillir quelques fragments de cet ouvrage, les tirant d'Eusèbe de Césarée, Jérôme, Augustin, Cyrille d'Alexandrie ${ }^{24}$. On sait que ces travaux n'ont jamais vu le jour, même s'il n'est pas difficile d'imaginer qu'au moins une partie de ces recherches fut par la suite refondue dans les Annotata ad Vetus Testamentum, à propos des Adiecta ad Danielem ${ }^{25}$.

Voilà pourquoi le mérite d'avoir attiré le premier l'attention des savants modernes sur les fragments qui restent de l'ouvrage anti-

20 ACO, III, 121, 24.

21 Enarr. in Joh. (PG, 123, 1141).

22 Ravennatis anonymi cosmographia, éd. M. Pinder et G. Parthney, Berlin, 1860, p. $91 ; 171 ; 176$.

23 Polycraticus, VII, 6 (PL, 199, 648 D).

24 Il s'agit du Ms. 1865 (XVIIe siècle), fol. $149 \mathrm{r}$ - $151 \mathrm{v}$, de la Bibliothèque Inguimbertine de Carpentras : Ex Porphyrio in Christianos collecta ab $\mathrm{H}$. Grotio.

25 En tout cas on ne trouve pas trace de travaux de Grotius sur Porphyre dans J.T. MeUlen - P.J.J. Diermanse, Bibliographie des écrits imprimés de Hugo Grotius, La Haye, 1950. 
chrétien de Porphyre revient au converti de Hambourg Lukas Holste (Holstenius). À la fin de sa De vita et scriptis Porphyrii philosophi dissertatio, publiée à Rome en $1630^{26}$, il tenta de décrire à grands traits le contenu de chaque livre, dans les limites des informations disponibles provenant des anciens polémistes ecclésiastiques qui avaient conservé les quelques fragments restants. Il croyait pouvoir reconstituer l'ouvrage de Porphyre de la façon suivante :

- le premier livre traitait des dissonances des Écritures Saintes et donc de leur origine simplement humaine, et des conflits qui avaient opposé entre eux même les deux principaux apôtres Pierre et Paul;

- le troisième livre avait pour thème l'interprétation de l'Écriture et condamnait la méthode de l'exégèse allégorique qu'avait pratiquée surtout Origène;

- le quatrième livre était au contraire consacré à l'histoire mosaïque et aux antiquités juives.

Pour les livres du cinquième au onzième, comme pour le deuxième d'ailleurs, Holste disait qu'il n'avait pas trouvé de citation à leur propos. Le douzième livre est le plus célèbre : dans ce livre Porphyre mène une violente polémique contre le livre de Daniel et ses prétendues prophéties, poursuivant la critique même dans le treizième livre.

En ajoutant d'autres fragments relatifs à la critique que Porphyre avait développée autour de l'histoire évangélique, Holste offrait une première reconstitution, forcément approximative et pleine de graves lacunes, du traité antichrétien de Porphyre.

Cette collection eut certainement un écho parmi les savants. Il est donc étonnant de constater que le bibliothécaire de Wolfenbüttel, Gotthold Ephraim Lessing, en polémiquant contre le pasteur Goeze, 150 ans après, regrette le fait qu'il n'ait pas survécu un seul fragment de l'ouvrage antichrétien de Porphyre et que soient irréparablement perdues même les trente - et plus - réfutations chrétiennes que Porphyre s'était bien attirées selon la Chronique de Dexter ${ }^{27}$. Plusieurs décennies après, Bernays eut beau jeu de démontrer que Lessing avait commis une double erreur, parce que d'un côté quelques fragments avaient tout de même survécu et que de l'autre la Chronique de Dexter,

26 Nous citons la dissertation de Lukas HoLsTe de la réimpression qui se trouve en appendice de A.J. FABRICIUs, Bibliotheca graeca, lib. IV, pars altera, Hamburgi, 1711, p. 207-281, en particulier les p. 273-281 : De Porphyrii libris contra Christianos.

27 Anti-Goeze, VI (Gesammelte Werke, éd. W. Stammler, München, 1959, I, p. 1094-1095). L'ouvrage polémique fut composé en 1778. 
que Lessing avait jugée authentique, devait être au contraire considérée comme un faux de l'époque moderne ${ }^{28}$.

Il fallut plus d'un siècle pour qu'un autre érudit, après Holste, s'aventurât dans l'entreprise ardue de présenter une nouvelle collection de fragments connus de cet ouvrage.

Le savant anglais Nathaniel Lardner publie son recueil des fragments antichrétiens de Porphyre à l'intérieur d'une collection plus vaste de témoignages païens sur la crédibilité de l'histoire de l'Évangile ${ }^{29}$. Il commence par le commentaire sur le fameux passage $\mathrm{du}$ troisième livre dans lequel Porphyre cite Origène pour critiquer rudement l'interprétation allégorique des Écritures en vogue chez les Chrétiens. La présentation suivante ne s'accorde pas avec l'ordre du traité porphyrien, mais bien avec celui des livres bibliques, d'abord l'Ancien, puis le Nouveau Testament, réservant surtout une grande place à la critique porphyrienne du livre de Daniel.

Il convient de signaler, au moins par simple curiosité érudite, le fait que dans cette savante dissertation Lardner se sert de beaucoup d'arguments pour démontrer l'inauthenticité de la Philosophie tirée des oracles, ouvrage habituellement attribué à Porphyre. Selon lui, cet ouvrage n'aurait pas pu être écrit par le païen Porphyre, vu que l'auteur parle d'une façon trop élogieuse du Christ, mais on devrait plutôt l'attribuer à un Chrétien ayant vécu après Constantin et qui aurait eu l'intention de critiquer d'une façon radicale la théologie et le culte du paganisme pour recommander à ses lecteurs précisément la religion chrétienne $\mathrm{e}^{30}$.

À part cette singulière interprétation de la Philosophie des oracles, destinée évidemment à ne pas avoir d'écho dans les études porphyriennes qui suivirent, il faut reconnaître que le recueil de Lardner ne pouvait être mieux mené, vu l'insuffisance des matériaux à disposition. Ce recueil devait donc rester pendant longtemps encore l'édition

28 J. BerNAYs, Theophrastos' Schrift über Frömmigkeit. Ein Beitrag zur Religionsgeschichte, Berlin, 1866, p. 133-134. En fait, la Chronique de Dexter qu'on lit dans la $P L, 31,49-574$, est une falsification d'un jésuite espagnol du XVIIe siècle.

29 N. LARDNer, The Credibility of the Gospel History, Part II : Testimonies of Ancient Heathens, Chap. XXXVII : Porphyry. Nous citons l'édition suivante : The Works of Nathaniel Lardner with a Life by Dr. Kippis, Vol. VII, Part II, London, 1838, p. 390-467, mais l'édition précédente portait la date de 1788.

30 Ibid., p. 450 sq. 
standard des fragments du traité antichrétien de Porphyre jusqu'au jour où quelque nouvelle découverte de textes inconnus et inédits viendrait changer substantiellement la situation ${ }^{31}$.

\section{Vers l'édition de Von Harnack}

La première moitié du XIXe siècle n'enregistre pas de progrès ni d'acquisitions nouvelles. Un article très intéressant de H. Kellner ${ }^{32}$ offre une bonne synthèse de tout ce que l'on aurait pu dire d'une façon documentée vers la moitié du siècle, de l'activité antichrétienne de Porphyre.

Il faut remarquer que Kellner, contrairement à Lardner (dont il semble ignorer la collection), comprit avec une intuition pénétrante que Porphyre avait élaboré sa polémique antichrétienne non seulement dans le traité en 15 livres mais aussi dans la Philosophie des oracles ${ }^{33}$. Cette observation, malheureusement, ne fut pas reprise et développée dans la bibliographie ultérieure qui suivit d'autres orientations; mais on comprend aisément, du moins selon l'esprit de l'escalier, qu'une étude parallèle des deux ouvrages, conduite de façon systématique, aurait certainement apporté des nouveautés significatives au débat.

Quoi qu'il en soit, les recherches ont tout de même connu un tournant décisif en 1876, lorsque par les soins de C. Blondel et P. Foucart, vit le jour l'Editio princeps d'un manuscrit athénien du XVe siècle contenant une grande partie de l'Apokritikos de Macaire de Magnésie ${ }^{34}$.

Cet ouvrage apologétique en forme de dialogue, qu'on peut dater du dernier quart du IVe siècle, rapporte les objections qu'un philosophe grec païen anonyme adresse contre la religion chrétienne et ses doctrines, en particulier contre les contradictions du Nouveau Testament. Après la publication de ce livre, les érudits se posèrent immédiatement la

31 Harnack, Porphyrius 'Gegen die Christen', p. 13, exprime un jugement favorable sur son devancier.

32 Der Neuplatoniker Porphyrius und sein Verhältnis zum Christentum, in ThQ, 47 (1865), p. 60-102.

33 Ibid., p. 80 sq.

34 Macarii Magnetis quae supersunt ex inedito codice edidit C. Blondel, Parisiis, 1876. Pour l'histoire de la tradition manuscrite de l'Apokritikos on se reportera au travail fondamental de G. SchalKHAUSSER, $Z u$ den Schriften des Makarios von Magnesia, Leipzig, 1907 (TU, 31/4), p. 5-113. 
question suivante : qui se cache derrière le païen anonyme ? Une longue discussion s'ensuivit, au cours de laquelle les noms les plus disparates furent proposés, de Sossianus Hiéroclès ${ }^{35}$ à Jamblique ${ }^{36}$ et à Julien l'Apostat ${ }^{37}$. Cependant c'est le nom de Porphyre qui recueillit les approbations les plus nombreuses et durables ${ }^{38}$.

Déjà avant la découverte de ce manuscrit athénien de Macaire, le théologien de Göttingen Magnus Crusius, au XVIIIe siècle, se basant sur les quelques renseignements qu'il détenait, avait avancé l'hypothèse qu'il fallait identifier à Porphyre le païen critiqué par Macaire ${ }^{39}$. Malgré l'incertitude exprimée par Pitra ${ }^{40}$, c'est précisément cette explication qui finit par s'imposer parmi les savants qui recommencèrent à analyser le problème à la fin du siècle dernier.

En publiant la première traduction allemande des objections du païen anonyme, Wagenmann identifiait ce dernier à Porphyre ${ }^{41}$, rencontrant tout de suite l'approbation de Neumann ${ }^{42}$. Quelques années plus tard, quatre autres études suivaient les mêmes traces. Il s'agit,

35 L. Duchesne, De Macario Magnete et scriptis eius, Parisiis, 1877, suivi par T.W. Crafer, Macarius Magnes, a Neglected Apologist, in JThS, 8 (1907), p. 401-423; 546-571.

36 J. Geffcken, Harnacks Ausgabe von Porphyrius 'Gegen die Christen', in Deutsche Literaturzeitung, 37 (1916), col. 1637-1642.

37 MöLLER, in Theologische Literaturzeitung, Nr. 19 (1877), p. 521-526; Th. ZAHN, $\mathrm{Zu}$ Makarius von Magnesia, in ZKG (1878), p. 523; plus récemment l'italien P. FrassineTTI, Sull'autore delle questioni pagane conservate nell'Apocritico di Macario di Magnesia, in Nuovo Didaskaleion, 3 (1949), p. 41-56.

38 La monographie de R. WAELKENS, L'économie, thème apologétique et principe herméneutique dans l' «Apocriticos" de Macarios Magnès, Louvain, 1974, est la meilleure introduction à l'œuvre de MACAIRE et à ses nombreux problèmes.

39 Il fut l'auteur de deux dissertaions sur MACAIRE DE MAGNÉSIE, datées de 1737 et 1745, qu'on peut lire aujourd'hui dans la Patrologia Graeca de Migne au tome $\mathrm{X}, 1343-1406$.

40 J.B. PITRA, Spicilegium Solesmense, I, Paris, 1852 (réimpr. Graz, 1962), p. 548.

41 Dans l'article : Porphyrius und die Fragmente eines Ungenannten in der athenischen Makariushandschrift, in Jahrbücher für deutsche Theologie, 23 (1878), p. 269-314.

42 C.I. NEUMANN, Iuliani imperatoris librorum contra Christianos quae supersunt, Lipsiae, 1880, p. 14-23. 
dans l'ordre chronologique, des contributions de Loesche ${ }^{43}$, Georgiades $^{44}$, Kleffner ${ }^{45}$ et Hauschildt ${ }^{46}$.

Tout compte fait, ces auteurs présentent l'ouvrage de Porphyre d'une manière identique. Quant à la disposition des fragments et leur interprétation, elles ne divergent pas du tout de celles précédemment illustrées par Holste et Lardner. La véritable nouveauté, très importante d'ailleurs, est que ces auteurs ont reconnu Porphyre comme l'adversaire critiqué par Macaire. Ainsi, ils proposèrent de combler la longue lacune des livres V-XI du traité antichrétien de Porphyre précisément avec les objections de l'anonyme de Macaire. Ils conclurent de cette façon que les sept livres, dont on ignorait tout auparavant, contenaient la réfutation de l'histoire de Jésus racontée dans les Évangiles et de l'enseignement même des apôtres.

On en était à ce point quand intervint Adolf von Harnack. Celui-ci consacra toute une monographie à l'étude des objections de l'Apokritikos de Macaire ${ }^{47}$, avec le propos délibéré de préparer un Baustein, une pierre de construction, pour son édition en projet du traité de Porphyre contre les Chrétiens ${ }^{48}$.

Nous ne voulons pas ici nous étendre sur le développement de la thèse de Harnack. Il nous suffit de dire que pour lui Macaire critique un traité qu'un païen a écrit contre le christianisme et que ce traité serait à son tour un Excerptum anonyme tiré de l'ouvrage antichrétien de Porphyre vers l'an 300 après J.-C. Macaire aurait donc combattu un adversaire qui avait résumé le traité de Porphyre, sans pour autant savoir que derrière son ennemi se trouvait précisément l'ouvrage de Porphyre!

Harnack allait produire son édition des fragments de Porphyre, plus d'un siècle après celle de Lardner, se fondant sur son travail préalable sur Macaire et la critique du philosophe païen. En 1916, à Berlin, fut

43 G. Loesche, Haben die späteren Neuplatonischen Polemiker gegen das Christenthum das Werk des Celsus benutzt?, in ZwissTh, 27 (1883), p. 257-302.

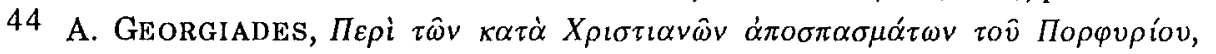
Leipzig, 1891 (dissertation en grec).

45 A.I. KlefFner, Porphyrius, der Neuplatoniker und Christenfeind, Paderborn, 1896, p. 42-97.

46 H. Hauschildt, De Porphyrio philosopho Macarii Magnetis apologetae

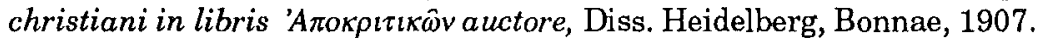

47 A. voN HARNACK, Kritik des Neuen Testaments von einem griechischen Philosophen des 3. Jahrhunderts (Die im Apocriticus des Macarius Magnes enthaltene Streitschrift), Leipzig, 1911 (TU, 37/4).

48 Kritik, p. 144. 
publiée la grande collection des témoignages et des fragments du traité de Porphyre contre les Chrétiens. Dans cette collection Harnack présentait d'une manière complète tous les problèmes critiques relatifs à cet ouvrage et illustrait les critères qui avaient présidé à la rédaction de sa nouvelle édition 49 .

En effet, cette édition de Harnack se caractérise essentiellement par deux aspects méthodologiques particuliers.

En premier lieu, Harnack, qui avait précédemment rapporté les objections de l'anonyme de Macaire, du moins indirectement, au traité perdu de Porphyre, se permit de faire des objections païennes une partie intégrante de son édition avec pour résultat que pas moins de 51 fragments sur 97 , plus de la moitié donc, sont constitués par ces mêmes objections.

Deuxièmement, Harnack adopta des critères tout à fait nouveaux dans la disposition des textes. D'un côté il estimait que c'était une entreprise désespérée que de vouloir reconstituer le contenu de chaque livre, à la manière traditionnelle, mais de l'autre il refusait même l'ordre choisi par Lardner qui consistait à disposer les fragments selon la succession des livres bibliques. C'est ainsi que Harnack décida de partager les documents en cinq grandes sections : 1) critique des évangélistes et des apôtres comme base de la critique générale du christianisme; 2) critique de l'Ancien Testament; 3) critique des actions et des paroles de Jésus; 4) critique du dogme chrétien; 5) critique de la situation ecclésiastique contemporaine ${ }^{50}$.

Comme introduction générale, Harnack choisit un passage du premier livre de la Praeparatio evangelica d'Eusèbe de Césarée dans lequel Wilamowitz avait déjà entrevu la présence de critiques contre le christianisme qui ne pouvaient venir que de Porphyre 51 .

Quelques années plus tard, Harnack publiait cinq nouveaux fragments latins tirés de l'ouvrage d'un certain rhéteur Pacatus contre Porphyre et transmis par une chaîne exégétique sur les quatre évangiles ${ }^{52}$.

49 Porphyrius 'Gegen die Christen', cité supra, n. 6.

50 Ibid., p. 3-22.

51 Il s'agit de Praep. evang., I, 2, 1-4, étudié par U. voN WILAMowitzMoellendorfF, Ein Bruchstück aus der Schrift des Porphyrius gegen die Christen, in ZNTW, 1 (1900), p. 101-105.

52 A. von Harnack, Neue Fragmente des Werks des Porphyrius gegen die Christen. Die Pseudo-Polycarpiana und die Schrift des Rhetors Pacatus gegen Porphyrius, in Sitzungsberichte der Preussischen Akademie der Wissen- 
L'édition des fragments de Porphyre établie par Harnack devait donc rester l'ouvrage de référence essentiel pour toute la discussion postérieure. En effet, tous ceux qui ont écrit sur ce sujet, pour un motif ou pour un autre, ont eu affaire à cet ouvrage. Mais à une période d'admiration inconditionnelle et d'acceptation de l'édition de Harnack a succédé une époque, plus proche de nous dans le temps, dans laquelle on a vu se multiplier les critiques et les réserves. Pour se rendre compte de la situation actuelle, il est nécessaire de dresser un tableau précis des développements de la question au cours de ce siècle; c'est seulement ainsi que l'on pourra continuer l'enquête et peut-être arriver à quelque chose de vraiment nouveau.

\section{L'après Harnarck : de l'approbation aux critiques}

Celui qui parcourt toute la bibliographie de notre siècle pourra facilement remarquer que l'ouvrage de Harnack a pendant longtemps réussi à s'imposer, grâce à la force de son érudition et à sa structure rigoureusement systématique, comme l'édition définitive, au-delà de laquelle il aurait même été impossible de s'aventurer.

Bidez déjà, dans sa biographie encore valable de Porphyre, avait accepté sans réserve l'identification de l'anonyme de Macaire avec Porphyre, selon les indications de Harnack ${ }^{53}$, rejetant les timides critiques qu'avait avancées Geffcken ${ }^{54}$.

Par la suite, un bon nombre de noms prestigieux suivit sans condition la thèse de Harnack, plaçant ainsi son ouvrage à la base de toute recherche historique et littéraire sur la question. Pour ne citer que les plus fameux, rappelons ici les noms de Moffatt ${ }^{55}$, de Labriolle ${ }^{56}$,

schaften, 1921, p. 266-284; 834-835. Cf. P. COURCELLE, Les lettres grecques en Occident de Macrobe à Cassiodore, Paris, 19482, p. 211-212.

53 J. BIDEz, Vie de Porphyre, le philosophe néo-platonicien avec les fragments des

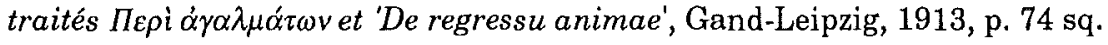

54 J. Gefrcken, Zwei griechische Apologeten, Leipzig-Berlin, 1907 (réimpr. Hildesheim-New York, 1970), p. 302 sq. Les travaux de BIDEz et HARNACK furent ensuite vulgarisés par L. VaGanaY, Porphyre, in DTC, XII/2 (Paris, 1935), col. 2555-2590.

55 J. MofFaTT, Great Attacks on Christianity, II : Porphyry 'Against Christians', in Expository Times, 43 (1931/32), p. 72-78.

56 P. DE LABRIOLLe, La réaction paienne, cité supra, n. 18, p. 223-296. 
Nestle ${ }^{57}$, Pierre Benoit ${ }^{58}$, Beutler ${ }^{59}$, Laurin ${ }^{60}$, Scheidweiler ${ }^{61}$, Schröder ${ }^{62}$, Anastos ${ }^{63}$, Gigon ${ }^{64}$, Démarolle ${ }^{65}$.

Il semblait donc que Harnack avait réussi à faire complètement oublier tout le long travail des siècles qui l'avaient précédé. Quelques tentatives de récupérer le plan originel de l'ouvrage de Porphyre, dans sa structure et ses contenus, comme celles opérées par $\mathrm{Crafer}^{66}$ et Hulen ${ }^{67}$, restèrent isolées et sans aucun succès appréciable. Dans toute la bibliographie relative au thème de la polémique pagano-chrétienne de l'antiquité tardive, l'ouvrage de Harnack avait pratiquement acquis le monopole incontesté à tel point que le seul progrès imaginable consistait à découvrir éventuellement, dans on ne sait quel ouvrage antique ou oriental, quelques nouveaux fragments à même d'enrichir la collection de Harnack.

En effet, des tentatives d'identifier quelques nouveaux textes de Porphyre ne manquèrent pas. En 1930, Jacoby ajouta à la collection de Harnack de nouveaux passages tirés du commentaire de Jérôme au

57 W. NESTLE, Die Haupteinwände des antiken Denkens gegen das Christentum, in $A R W, 37$ (1941), p. 51-100.

58 Pierre BENOIT, Un adversaire du Christianisme au IIIe siècle : Porphyre, in RevBib, 54 (1947), p. 543-572 (réimpr. dans Exégèse et Théologie, II, Paris, 1961 , p. 415-447).

59 R. Beutler, Porphyrios, in RE, XXII 1 (1953), c. 275-313, 298 sq.

60 J.-R. LAURIN, Orientations maîtresses des apologistes chrétiens de 270 à 361 , Romae, 1954 (Analecta Gregoriana, 61), p. 37-52.

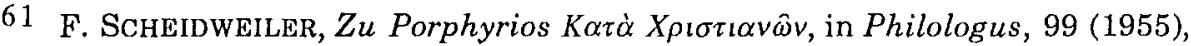
p. 304-312.

62 H.O. SCHRöDer, Celsus und Porphyrius als Christengegner, in Die Welt als Geschichte, 17 (1957), p. 190-202.

63 M.W. ANAstos, Porphyry's Attack on the Bible, in The Classical Tradition. Literary and Historical Studies in Honour of H. Caplan, Ithaca N.Y., 1966, p. 421-451.

64 Olof Gigon, Die antike Kultur und das Christentum, Gütersloh, 1966, p. 118 122.

65 J.-M. DÉmarolle, La Chrétienté à la fin du IIIe s. et Porphyre, in GRBS, 12 (1971), p. 49-57; EAD., Un aspect de la polémique paienne à la fin du IIIe siècle : le vocabulaire chrétien de Porphyre, in VigChr, 26 (1972), p. 117-129.

66 T.W. CRAFER, The Work of Porphyry against the Christians and its Reconstruction, in JThS, 15 (1914), p. 360-395; 481-512.

67 A.B. Hulen, Porphyry's Work against the Christians : an Interpretation, Scottdale, 1933 (Yale Studies in Religion, 1), p. 45 sq. 
livre de Daniel, en les attribuant directement à Porphyre ${ }^{68}$. Pierre Nautin indiqua en 1950 trois nouveaux fragments du traité antichrétien de Porphyre dans le premier livre de la Praeparatio evangelica d'Eusèbe de Césarée ${ }^{69}$, alors que Franz Altheim et Ruth Stiehl allèrent en chercher jusque dans des sources arabes et syriaques ${ }^{70}$. Les papyrus de Toura, à leur tour, ont rendu une partie considérable des commentaires bibliques de Didyme l'Aveugle qui font état d'opinions de Porphyre tirées certainement de son ouvrage antichrétien ${ }^{71}$. Encore récemment Brian Croke a proposé de faire rentrer dans le XIIe livre du traité antichrétien les fragments de la Chronique de Porphyre, dont il met l'existence en doute avec des bons arguments ${ }^{72}$. On pourrait continuer ainsi.

Cependant, tout ce travail d'érudition n'a amené rien de vraiment nouveau, dans le sens qu'il n'a pas contribué à surmonter les difficultés méthodologiques que présente toute tentative d'aboutir à une nouvelle édition critiquement fondée du traité antichrétien de Porphyre.

Pour ce motif, nous jugeons que sont plus importantes les contributions qui, sans apporter de nouvelles découvertes ou attributions de textes, ont cependant ouvert la voie à la compréhension des limites objectives de la collection de Harnack et ont fini par remettre en question ses prémisses.

Devancé par les observations subtiles de deux savants italiens, Paolo Frassinetti ${ }^{73}$ et Sosio Pezzella ${ }^{74}$, l'historien Timothy Barnes

68 F. JACOBY, Die Fragmente der Griechischen Historiker (FGrHist), Berlin, 1930, II B, 260, p. 1221-1229; II D, p. 877-884.

69 P. Nautin, Trois autres fragments du livre de Porphyre 'Contre les Chrétiens', in RevBib, 57 (1950), p. 409-416.

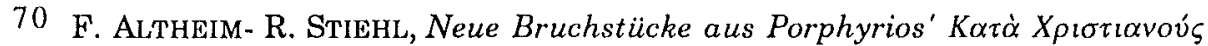
(sic !), in Gedenkschrift für G. Rohde. A ПAPXAI. Untersuchungen zur klassischen Philologie und Geschichte des Altertums, 4 (1961), p. 23-38.

71 Voir à ce propos les études de D. HAGEDorN-R. MERKELBACH, Ein neues Fragment aus Porphyrios gegen die Christen, in VigChr, 20 (1966), p. 86-90; G. BINDER, Eine Polemik des Porphyrios gegen die allegorische Auslegung des Alten Testaments durch die Christen, in ZPE, 3 (1968), p. 81-95; la note de M. GRONEWALD, ibid., p. 96; et récemment l'article de Ph. Sellew, Achilles or Christ? Porphyry and Didymus in Debate over Allegorical Interpretation, in $H T h R, 82$ (1989), p. 79-100.

72 Brian Croke, Porphyry's Anti-Christian Chronology, in JThS, 34 (1983), p. 168-185.

73 P. FrassinetTi, Sull'autore delle questioni pagane, cité supra, n. 37.

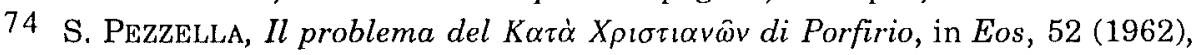
p. 87-104. 
lançait en 1973 une attaque directe contre l'édition de Harnack qui a justement marqué une date ${ }^{75}$.

Barnes donne une double contribution. En ce qui concerne l'attribution des fragments, il soutient fermement qu'en aucun cas on ne peut affirmer que Macaire de Magnésie préserve tels quels les mots et les formulations précises de Porphyre. Il serait donc plus sage de ne pas tenir compte de Macaire pour arriver à des résultats valables sur le traité antichrétien de Porphyre et porter plutôt l'attention sur les fragments que les écrivains ecclésiastiques postérieurs attribuent à Porphyre - en le nommant - d'une manière explicite et non ambiguë 76 .

Barnes tend à situer la date de composition du traité dans les années à cheval entre les IIIe et IVe siècles, en liaison étroite avec la persécution de Dioclétien ${ }^{77}$.

Nous ne nous attarderons pas pour le moment sur le problème extrêmement complexe de la datation du traité de Porphyre et sur la variété des argumentations historiques et littéraires qu'on peut avancer pour défendre l'une ou l'autre opinion. Ce qui nous intéresse, c'est plutôt de mettre en évidence la force révolutionnaire qui se trouve dans l'essai de Barnes : il a en effet réouvert la discussion qui semblait figée à jamais sur les positions de Harnack.

À la fin d'une étude dans laquelle il se demandait : «Où en est la collecte des fragments ?», André Benoît notait il y a quelques années que ce qui avait été élaboré dans l'édition de Harnack n'était qu'un "plan arbitraire et mal justifié» et qu'il s'agissait aussi d'«un classement parfaitement arbitraire des fragments" ${ }^{78}$. Ces affirmations sont difficilement imaginables avant ou sans l'apport de Barnes. Benoît aussi, d'ailleurs, devait reconnaître que les fragments reproduits par Macaire de Magnésie pouvaient tout au plus, après un examen critique, rapporter des thèmes d'origine porphyrienne, mais non les paroles mêmes du philosophe néoplatonicien ${ }^{79}$. Et Benoît de conclure, sur un ton peut-être excessivement optimiste : "Ainsi la

75 T.D. BARNES, Porphyry 'Against the Christians' : Date and the Attribution of Fragments, in JThS, 24 (1973), p. 424-442.

76 Ibid, p. 430.

77 Ibid, p. 433 sq.

78 A. Benoît, Le "Contra Christianos" de Porphyre : où en est la collecte des fragments ?, in Paganisme, Judaïsme, Christianisme. Influences et affrontements dans le monde antique. Mélanges offerts à Marcel Simon, Paris, 1978, p. 261-275, 266.

79 Ibid., p. 269 sq. 
collection proposée par Harnack n'est pas close, elle s'est augmentée... et elle est susceptible de continuer à s'augmenter...» 80 .

Dans une importante contribution publiée en 1980 dans Aufstieg und Niedergang der Römischen Welt, A. Meredith se déclare pleinement d'accord avec la critique radicale de Barnes ${ }^{81}$. Une fois écartés les fragments tirés de l'Apokritikos de Macaire de Magnésie, Meredith affirme donc que seuls 46 des 97 fragments collectionnés par Harnack peuvent être attribués avec quelque probabilité à l'ouvrage perdu de Porphyre. Mais Meredith avertit d'une manière hypercritique que, même avec un nombre aussi réduit, il est impossible d'être sûr de l'authenticité de ces fragments et de leur provenance du traité antichrétien, parce que même plusieurs fragments qui ne dérivent pas de Macaire font, à leur tour, l'objet d'un doute ${ }^{82}$.

Selon Meredith, une édition du traité porphyrien compterait en tout 46 fragments, dont 22 proviennent de Jérôme, 5 d'Augustin, 7 d'Eusèbe, plus 12 autres de diverses sources (Épiphane, Théodoret, Théophylacte).

Tout en réduisant d'une façon drastique les documents disponibles, les observations critiques de Barnes ont pourtant contribué à faire évaluer avec plus de réalisme la possibilité de parvenir à une édition significative de l'ouvrage de Porphyre, à moins qu'elle ne soit enrichie par de nouvelles découvertes inattendues.

L'Américain R. Wilken, dernier auteur dans le temps à s'être penché sur notre problème, accueillant lui aussi les critiques de Barnes, est amené à conclure, à regret, que, malgré tout, sans les fragments de Macaire, notre connaissance de l'ouvrage porphyrien devient extrêmement approximative ${ }^{83}$. Wilken, en outre, attire l'attention sur le fait - déjà remarqué par Kellner et d'autres - qu'à part le traité contre les Chrétiens en quinze livres, la polémique antichrétienne de Porphyre a pu être explicitée, bien que sous une autre forme, dans un autre ouvrage de Porphyre, la Philosophie des oracles, en trois livres 84 .

80 Ibid., p. 268.

81 Anthony MEREDITH, Porphyry and Julian against the Christians, in ANRW, II, 23.2, Berlin-New-York, 1980, p. 1119-1149, pour PORPHYRE, p. 1125-1137.

82 Ibid., p. 1127-1128.

83 R.L. Wilken, The Christians as the Romans Saw Them, New Haven-London, 1984, p. 126-163; 135-136.

84 Ibid., p. 137 sq.; 148 sq. WILKEN avait déjà abordé la question pour la première fois dans l'article Pagan Criticism of Christianity: Greek Religion and Christian Faith, in Early Christian Literature and the Classical Intellectual Tradition in honorem R.M. Grant, Paris, 1979 (Théologie Historique, 53), p. 117-134; 129 sq. 
Cette idée que Porphyre aurait écrit deux ouvrages, et non un seul, contre les Chrétiens, nous voyons qu'elle est maintenant partagée par Frend ${ }^{85}$ et Droge ${ }^{86}$. En réalité, il faut admettre que les rapports entre la Philosophie des oracles et le traité contre les Chrétiens mériteraient certainement d'être étudiés d'une manière plus précise et plus détaillée qu'on ne l'a fait jusqu'à présent. Sur cette voie, on peut s'attendre raisonnablement à des découvertes surprenantes.

\section{Que faire ? Propositions et hypothèses pour poursuivre les recherches}

Au terme de ce bilan rapide des événements qui pendant un millénaire et demi ont marqué le destin singulier du traité antichrétien de Porphyre, on se rend vite compte que les difficultés sont encore nombreuses et complexes.

Nous devons signaler au moins deux problèmes dont la solution nous semble préliminaire à toute tentative de réexaminer la question en vue d'une future édition - si possible - des fragments conservés de l'ouvrage.

Pour commencer, les objections païennes que rapporte Macaire de Magnésie. Désormais la confiance que Harnack avait mise en elles pour récupérer plus de la moitié des fragments de son édition, a connu une crise irréversible; il ne reste donc plus qu'à prendre acte de la nécessité de renoncer une fois pour toutes à l'apport qu'on aurait pu attendre de ces objections.

Nous sommes nous aussi de l'avis que, quel que soit celui qui se cache derrière l'anonyme de Macaire, dans tous les cas il ne peut pas s'agir de Porphyre. C'est justement son nom qu'il faut exclure puisque Macaire, à un certain point dans les solutiones, renvoie son interlocuteur anonyme à l'ouvrage de Porphyre La philosophie des oracles :

$\mathrm{Tu}$ peux vérifier ces choses dans la «Philosophie des oracles» et apprendre soigneusement la liste des choses sacrifiées, si tu lis l'oracle d'Apollon sur les sacrifices que Porphyre, plein d'orgueil, livra en secret à ses disciples, en les chargeant du terrible serment -

85 W.H.C. FREND, Prelude to the Great Persecution: the Propaganda War, in JEH, 38 (1987), p. 1-18.

86 A.J. DRoge, Homer or Moses? Early Christian Interpretations of the History of Culture, Tübingen, 1989 (Hermeneutische Untersuchungen zur Theologie, 26), p. 171-180. 
comme lui-même le jugeait - de ne pas divulguer sans retenue ces choses aux profanes 87 .

Nous ne savons pas si Macaire avait lu cette œuvre de Porphyre en entier. Il aurait pu en effet citer ce passage de la Philosophie des oracles après l'avoir tiré de la Praeparatio evangelica d'Eusèbe de Césarée ${ }^{88}$. En tout cas, cette citation, à elle seule, suffit pour démontrer que Macaire connaissait Porphyre comme une personne bien distincte de son adversaire. C'est donc en vain que Harnack essaya de contourner l'obstacle en ayant recours à l'hypothèse, tout à fait arbitraire, que Macaire aurait utilisé un extrait du traité antichrétien de Porphyre qui l'aurait empêché de reconnaître la vraie identité de la source païenne qu'il réfutait ${ }^{89}$.

D'ailleurs, il est impossible d'identifier l'objecteur païen à Porphyre, vu qu'il se situe clairement vers les années soixante du IVe siècle 90 .

En définitive, la seule chose, semble-t-il, que l'on peut admettre, c'est que dans les objections de l'anonyme se trouvent des critiques d'inspiration porphyrienne, à côté d'autres thèmes qui sont traités par Hiéroclès ou Julien l'Apostat. Mais rien de plus. Comme l'a remarqué justement Goulet : «Il est donc fort imprudent d'insérer les objections du Monogenés parmi les fragments du traité de Porphyre même si elles nous transmettent des arguments authentiques de cet ouvrage». Et il ajoute en note : «À elle seule, cette conclusion impose une remise en question de la reconstitution du Contra Christianos entreprise par A. von Harnack» 91 .

L'autre problème, jusqu'ici sans réponse, consiste à savoir de quels sujets pouvaient traiter les livres II et XV, et surtout les livres du V au XI. Tout le monde sait qu'aucune citation n'a été tirée de ces livres, qu'elle soit directe ou indirecte, et cela ne peut que susciter la surprise ou des

87 MaCAIRE, Apokritikos, III, 42 (p. 145-146, Blondel). haurienda librorum reliquiae, Berlin, 1856 (réimpr. Hildesheim, 1962), p. 111 sq., ne connaissait pas encore MACAIRE!

89 A. vON HARNACK, Kritik des Neuen Testaments, p. 137 sq.

90 Cf. Apokritikos, IV, 2 et IV, 5. Ces données chronologiques sont confirmées par NicÉPhore de Constantinople, Antirrhetica, VIII (éd. J.B. Pitra, Spic. Solesm, I, p. 307).

91 R. GouLET, La théologie de Makarios Magnès, in MSR, 34 (1977), p. 45-69; 145180 , p. 46 et note 2. De GoULET on peut voir aussi l'article Porphyre et Macaire de Magnésie, in Studia Patristica, XV, Berlin, 1984 (TU, 128), p. 448-452. 
soupçons. C'est là, à notre avis, que s'ouvre un domaine de recherches certainement plus prometteur que celui qui concerne les objections de l'anonyme de Macaire.

Il faut, en effet, reconnaître honnêtement que la recherche d'éventuelles allusions au traité antichrétien de Porphyre dans la littérature patristique n'a rien apporté, du moins jusqu'à aujourd'hui, de vraiment solide. Des ouvrages tels que l'Apokritikos de Macaire ou bien les Quaestiones Veteris et Novi Testamenti de l'Ambrosiaster ${ }^{92}$ ne peuvent rien offrir de sûr. Et il ne semble pas qu'on puisse beaucoup attendre d'une exploration désespérée des chaînes exégétiques et de la littérature des Quaestiones et responsiones, qui pourtant sont très riches en objections et critiques bibliques d'inspiration païenne. Si l'on part du présupposé, évidemment sans fondement, que toutes les objections et les critiques contre la Bible et contre la doctrine chrétienne doivent d'une certaine manière remonter à Porphyre, jusqu'à quel point ne se sentira$\mathrm{t}$-on pas en droit d'arriver ? À suivre cette méthode de recherche on court le risque de se fourvoyer dans le sens que, par cette voie, on n'arrivera jamais - me semble-t-il - à la certitude d'avoir mis la main sur des textes porphyriens authentiques.

D'autre part, le manque de vérifications objectives et surtout l'absence du nom de Porphyre nous empêchent d'utiliser pour l'édition de ses fragments antichrétiens même les textes patristiques dans lesquels nous avons facilement indiqué comme cible principale l'ouvrage contre les Chrétiens. Je me réfère précisément à la polémique contre les viri novi dans l'Adversus Nationes d'Arnobe de Sicca ${ }^{93}$ et à l'apologie d'Athanase d'Alexandrie Contre les païens sur l'Incarnation du Verbe 94.

On peut difficilement contester, à ce point, que pour des exigences critiques, auxquelles on ne peut pas renoncer, le choix s'impose de se fier uniquement aux citations textuelles ou littérales du traité de

92 Contrairement à ce que pensait $P$. CoURCELle, Critiques exégétiques et arguments antichrétiens rapportés par Ambrosiaster, in VigChr, 13 (1959), p. 133-169.

93 Voir à ce propos mon article Un oracle antichrétien chez Arnobe, in Mémorial Dom Jean Gribomont (1920-1986), Roma, 1988, p. 107-129.

94 J'ai traité cette question dans ma contribution La Croix et les idoles d'après l'apologie d'Athanase 'Contre les païens', in A. Gonzalez Blanco (éd.), Aculturaciòn y Cristianismo en tiempos del Imperio Romano, Murcia, 1990 (Antigüedad y Cristianismo, vol. VII), à paraître. 
Porphyre. Tout au plus, pourra-t-on prendre en considération les citations indirectes dont le contenu est attribué, par les auteurs qui les rapportent, d'une manière explicite et non ambiguë, à Porphyre. Dans le cas contraire, on risquera inévitablement d'insérer dans le corpus des fragments non seulement des dubia, mais, pire encore, des spuria!

En fait, à part la découverte possible et souhaitable de nouveaux fragments de l'ouvrage antichrétien de Porphyre, nous croyons qu'il y a encore beaucoup de travail à faire pour collectionner d'une façon systématique toutes les citations, directes et indirectes, de Porphyre présentes dans la documentation patristique : opération que ni Harnack ni tous ceux qui l'ont suivi n'ont encore réussi à conclure.

À quels résultats pourra nous amener la lecture systématique et élargie des textes patristiques dans lesquels est mentionné l'ouvrage antichrétien de Porphyre ? Il est difficile de le dire pour le moment. Au fond, il s'agit d'une exploration aventureuse qui peut réserver de grosses surprises.

Nous disions en commençant que le traité de Porphyre contre les Chrétiens est un objet mystérieux puisque nous en ignorons presque tout, la date de composition, la structure, le contenu de chaque livre, bien qu'on ait commencé les recherches il y a plus de trois siècles et demi ! Nous nous demandons si, pour sortir de cette fâcheuse impasse, il n'est pas nécessaire d'abandonner les voies qu'a suivies jusqu'à présent la bibliographie traditionnelle - qui n'a aboutit à rien, même si elle est éprouvée et rassurante - et de répartir en posant de nouvelles questions qui supposent un contact direct avec les textes anciens.

Pas même le titre de l'œuvre de Porphyre ne peut échapper à ce processus qui remet tout en question.

Au terme d'une étude conduite pour dévoiler la nature et l'auteur des Libri platonicorum, dont la lecture mena Augustin à abandonner le manichéisme et à se convertir au catholicisme, nous avons avancé l'hypothèse que de tels livres devraient être identifiés à la Philosophie des oracles de Porphyre, traduite en latin par Marius Victorinus vers la moitié du IVe siècle. Augustin ne l'aurait pas déclaré publiquement dans les Confessions, se réfugiant dans l'appellation vague et approximative de Libri platonicorum, pour la raison que la Philosophie des oracles, à son tour, ne serait rien d'autre que le traité antichrétien de Porphyre ${ }^{95}$.

95 'Quosdam Platonicorum libros'. The Platonic Readings of Augustine in Milan, in VigChr, 43 (1989), p. 248-281. 
Dans le cas où l'on réussirait à confirmer une telle hypothèse grâce à la confrontation avec une documentation plus vaste, on relancerait toute la discussion relative, non seulement à l'identité de l'ouvrage antichrétien de Porphyre, à sa structure et à sa composition, mais aussi à l'évolution religieuse et intellectuelle de l'auteur. Bien difficile de prévoir à quels résultats peut aboutir une telle opération!

Via Pietro Metastasio, 16

Pier Franco BEATRICE

I - 35125 PADOVA

\section{Liste des abréviations}

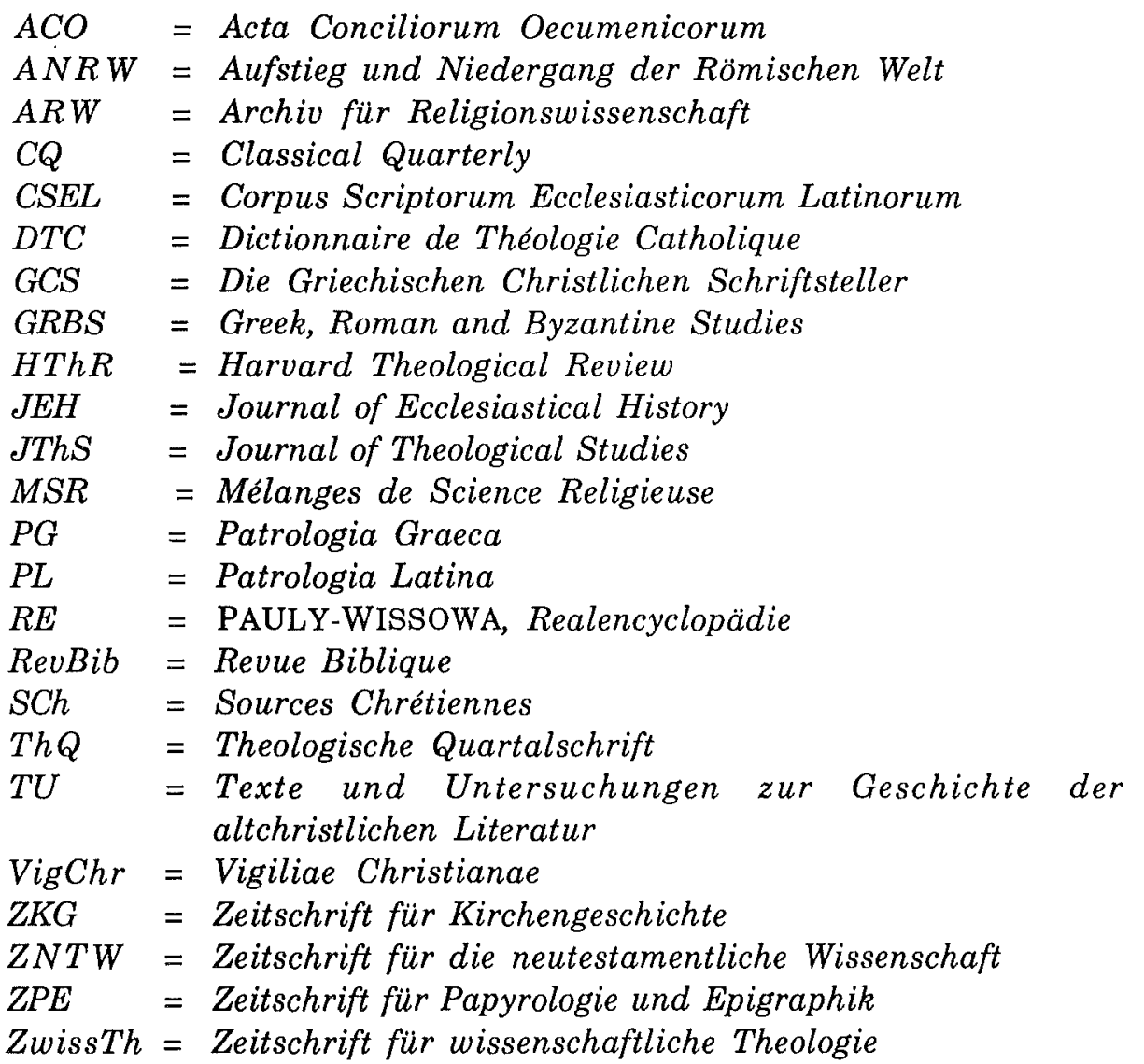

